\title{
Correction to: Determinants of healthcare seeking and out-of-pocket expenditures in a "free" healthcare system: evidence from rural Malawi
}

Meike Irene Nakovics ${ }^{1 *}$, Stephan Brenner ${ }^{1}$, Grace Bongololo $^{2}$, Jobiba Chinkhumba ${ }^{3}$, Olivier Kalmus ${ }^{1}$, Gerald Leppert ${ }^{4}$ and Manuela De Allegri ${ }^{1}$

\section{Correction to: Health Econ Rev 10, 14 (2020) \\ https://doi.org/10.1186/s13561-020-00271-2}

Following publication of the original article [1], the authors reported that the number "37.01" in Table 2 was moved up so it is now in the wrong line. The correct Table 2 is shown below. The original article [1] has been updated.

\footnotetext{
Author details

${ }^{1}$ Heidelberg Institute of Global Health, Faculty of Medicine and University Hospital, University of Heidelberg, Heidelberg, Germany. ${ }^{2}$ Research for Equity and Community Health (REACH) Trust, Lilongwe, Malawi. ${ }^{3}$ University of Malawi College of Medicine, BlantyreSouthern RegionMalawi. ${ }^{4}$ German Institute for Development Evaluation (DEval), Bonn, Germany.
}

Published online: 01 July 2020

\section{Reference}

1. Nakovics MI, Brenner S, Bongololo G, et al. Determinants of healthcare seeking and out-of-pocket expenditures in a "free" healthcare system: evidence from rural Malawi. Health Econ Rev. 2020;10:14 https://doi.org/10. 1186/s13561-020-00271-2.

\footnotetext{
The original article can be found online at https://doi.org/10.1186/s13561020-00271-2.

*Correspondence: nakovics@web.de

The original article can be found online at https://doi.org/10.1186/s13561020-00271-2

${ }^{1}$ Heidelberg Institute of Global Health, Faculty of Medicine and University Hospital, University of Heidelberg, Heidelberg, Germany
}

(c) The Author(s). 2020 Open Access This article is licensed under a Creative Commons Attribution 4.0 International License, which permits use, sharing, adaptation, distribution and reproduction in any medium or format, as long as you give appropriate credit to the original author(s) and the source, provide a link to the Creative Commons licence, and indicate if changes were made. The images or other third party material in this article are included in the article's Creative Commons licence, unless indicated otherwise in a credit line to the material. If material is not included in the article's Creative Commons licence and your intended use is not permitted by statutory regulation or exceeds the permitted use, you will need to obtain permission directly from the copyright holder. To view a copy of this licence, visit http://creativecommons.org/licenses/by/4.0/ The Creative Commons Public Domain Dedication waiver (http://creativecommons.org/publicdomain/zero/1.0/) applies to the data made available in this article, unless otherwise stated in a credit line to the data. 
Table 2 Sample characteristics

\begin{tabular}{|c|c|c|c|c|c|c|c|}
\hline & & \multicolumn{2}{|c|}{$\begin{array}{l}\text { Acute ill } \\
\text { sample } \\
(n=2740)\end{array}$} & \multicolumn{2}{|c|}{$\begin{array}{l}\text { Utilization formal } \\
\text { care sample } \\
(n=1884)\end{array}$} & \multicolumn{2}{|c|}{$\begin{array}{l}\text { Positive out-of-pocket } \\
\text { expenditures on } \\
\text { medical treatment } \\
\text { sample } \\
(n=494)\end{array}$} \\
\hline \multirow[t]{3}{*}{ Sample scale } & & \multicolumn{2}{|l|}{$\mathbf{N}$} & \multicolumn{2}{|l|}{$\mathbf{N}$} & \multicolumn{2}{|l|}{$\mathbf{N}$} \\
\hline & Number of villages & \multicolumn{2}{|l|}{101} & \multicolumn{2}{|l|}{98} & \multicolumn{2}{|l|}{75} \\
\hline & Number of households & \multicolumn{2}{|l|}{1037} & \multicolumn{2}{|l|}{718} & \multicolumn{2}{|l|}{186} \\
\hline \multicolumn{8}{|l|}{ Individual level } \\
\hline & & Mean & SD & Mean & SD & Mean & SD \\
\hline Distance to the closest health facility $(\mathrm{km})$ & continuous & 2.22 & 1.25 & 2.19 & 1.24 & 2.14 & 1.28 \\
\hline Age (years) & continuous & 21.43 & 18.2 & 19.56 & 17.57 & 19.62 & 17.12 \\
\hline Illness duration (days] & continuous & 7.91 & 8.27 & 8.67 & 8.85 & 9.58 & 10.21 \\
\hline \multirow[t]{2}{*}{ Accompanying persons } & continuous & & & 1.19 & 0.61 & 1.22 & 0.60 \\
\hline & & $\mathbf{N}$ & $\%$ & $\mathbf{N}$ & $\%$ & $\mathbf{N}$ & $\%$ \\
\hline \multirow[t]{4}{*}{ Age } & $0=0-4$ years & 513 & 18.72 & 419 & 22.24 & 117 & 23.68 \\
\hline & $1=5-14$ years & 793 & 28.94 & 562 & 29.83 & 133 & 26.92 \\
\hline & $2=15-39$ years & 971 & 35.44 & 637 & 33.81 & 171 & 34.62 \\
\hline & $3=39+$ years & 463 & 16.90 & 266 & 14.12 & 73 & 14.78 \\
\hline \multirow[t]{2}{*}{ Sex } & $0=$ male; & 1283 & 46.82 & 844 & 44.80 & 230 & 46.56 \\
\hline & 1 = female & 1457 & 53.18 & 1040 & 55.20 & 264 & 53.44 \\
\hline \multirow{2}{*}{$\begin{array}{l}\text { Education (education of household } \\
\text { head if child }<14 \text { years) }\end{array}$} & $0=$ no formal education & 1726 & 62.99 & 1164 & 61.78 & 295 & 59.72 \\
\hline & $1=$ any formal education & 1014 & 37.01 & 720 & 38.22 & 199 & 40.28 \\
\hline \multirow[t]{2}{*}{ Household head } & $0=$ others; & 2157 & 78.72 & 1523 & 80.84 & 391 & 79.15 \\
\hline & 1 = being household head & 583 & 21.28 & 361 & 19.16 & 103 & 20.85 \\
\hline \multirow[t]{2}{*}{ Reported chronic illness } & $0=$ no chronic illness reported; & 2356 & 85.99 & 1678 & 89.07 & 436 & 88.26 \\
\hline & $1=$ chronic illness reported & 384 & 14.01 & 206 & 10.93 & 58 & 11.74 \\
\hline \multirow[t]{2}{*}{ Limitation imposed on routine activities } & $0=$ no perceived limitation on routine activities & 1047 & 38.21 & 587 & 31.16 & 125 & 25.30 \\
\hline & $1=$ perceived limitation on routine activities & 1693 & 61.79 & 1297 & 68.84 & 369 & 74.70 \\
\hline \multirow[t]{2}{*}{ Hospitalization } & $0=$ no hospitalization; & 2636 & 96.20 & 1780 & 94.48 & 463 & 93.72 \\
\hline & $1=$ hospitalization & 104 & 3.80 & 104 & 5.52 & 31 & 6.28 \\
\hline \multirow[t]{4}{*}{ Socio economic status (wealth quartiles) } & $1=$ poorest (1st wealth quartile) & 686 & 25.04 & 484 & 25.69 & 108 & 21.86 \\
\hline & 2 = poor (2nd wealth quartile) & 685 & 25.00 & 486 & 25.80 & 109 & 22.06 \\
\hline & $3=$ less poor (3rd wealth quartile) & 685 & 25.00 & 478 & 25.37 & 126 & 25.51 \\
\hline & $4=$ least poor (4th wealth quartile) & 684 & 24.96 & 436 & 25.14 & 151 & 30.57 \\
\hline \multirow[t]{2}{*}{ Household size } & $0=0-5$ members; & 1483 & 54,12 & 1036 & 54.99 & 297 & 60.12 \\
\hline & $1=5+$ members & 1257 & 45,88 & 848 & 45.02 & 197 & 39.88 \\
\hline \multirow[t]{2}{*}{ Location of household } & $0=$ rural & 2598 & 94.82 & 1791 & 95.06 & 470 & 95.14 \\
\hline & $1=$ urban & 142 & 5.18 & 93 & 4.94 & 24 & 4.86 \\
\hline
\end{tabular}

SD Standard Deviation 